\title{
HLA-associated viral polymorphism in chronically HIV-1-infected Japanese cohort: analysis of four-digit HLA allele level
}

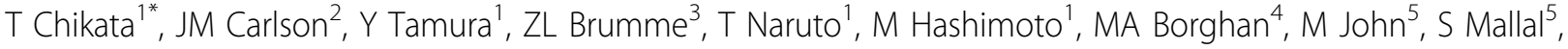 \\ H Gatanaga ${ }^{6}$, S Oka $^{6}$, M Takiguchi $^{1}$
}

From AIDS Vaccine 2012

Boston, MA, USA. 9-12 September 2012

\section{Background}

It is assumed that the difference of HLA class I distribution among ethnic populations influences HIV evolution because HLA-restricted immune pressure selects escape mutations. Approximately 50\% of HLA class I alleles are shared between Japanese and Caucasians. The analysis of HLA-associated polymorphism (HLA-AP) in both Japanese and Caucasian infected with clade $B$ virus is expected to clarify the difference of HIV-1 evolution between both populations.

\section{Methods}

We sequenced Gag, Pol, and Nef genes in 430 treatmentnaïve Japanese chronically infected with HIV-1 clade B and then identified HLA-associated amino acids at each codon using a phylogenetically corrected logistic regression model and false discovery rates to correct for multiple tests.

\section{Results}

We completely determined 400, 366, and 309 sequences of Gag, Pol, and Nef, respectively, and then analyzed polymorphisms associated with 78 four-digit HLA alleles (21 HLA-A, 38 HLA-B, and 19 HLA-C alleles). At the threshold of $\mathrm{q}<0.2$, we found 195 HLA-APs (67 in Gag, 61 in Pol, and 67 in Nef). These polymorphisms were observed at 39 of 501 (7.8\%) Gag, 42 of 1004 (4.2\%) Pol, and 33 of 207 (16.0\%) Nef codons. Ninety-six HLA-APs associated with more than one HLA subtype allele were detected in 4-digital HLA allele analysis. Approximately $40 \%$ of HLA-APs were associated with HLA alleles predominantly found in Asia. Out of HLA-APs associated with common HLA alleles, approximately $50 \%$ were found in Caucasian population (IHAC cohort).

\section{Conclusion}

This study demonstrated that only $30 \%$ of HLA-APs were shared between Caucasians and Japanese, indicating that the difference in HLA allele distributions resulted in distinct HIV-1 evolution.

\section{Author details \\ ${ }^{1}$ Center for AIDS Research, Kumamoto University, Kumamoto, Japan. ${ }^{2}$ eScience Group, Microsoft Research, Redmond, WA, USA. ${ }^{3}$ Faculty of Health Sciences, Simon Fraser University, Burnaby, British Columbia, Canada. ${ }^{4}$ College of Arts and Sciences, University of Nizwa, Oman. ${ }^{5}$ Royal Perth Hospital, Murdoch University, Perth, Australia. ${ }^{6}$ AIDS Clinical Center, National Center for Global Health and Medicine, Tokyo, Japan.}

Published: 13 September 2012

\section{doi:10.1186/1742-4690-9-S2-P269}

Cite this article as: Chikata et al:: HLA-associated viral polymorphism in chronically HIV-1-infected Japanese cohort: analysis of four-digit HLA allele level. Retrovirology 2012 9(Suppl 2):P269.

${ }^{1}$ Center for AIDS Research, Kumamoto University, Kumamoto, Japan

Full list of author information is available at the end of the article

(C) 2012 Chikata et al; licensee BioMed Central Ltd. This is an Open Access article distributed under the terms of the Creative Commons 